\title{
GLOBAL PHASE PORTRAITS FOR THE ABEL QUADRATIC POLYNOMIAL DIFFERENTIAL EQUATIONS OF SECOND KIND WITH $\mathbb{Z}_{2}$-SYMMETRIES
}

\author{
JAUME LLIBRE $^{1}$ AND CLAUDIA VALLS ${ }^{2}$
}

\begin{abstract}
We provide normal forms and the global phase portraits in the Poincaré disk for all Abel quadratic polynomial differential equations of the second kind with $\mathbb{Z}_{2}$-symmetries.
\end{abstract}

\section{Introduction AND STATEMENT OF THE MAIN RESUlts}

There are more than one thousand papers published in quadratic polynomial differential systems (or simply quadratic systems). The difficulty of studying these differential systems is due to the fact that they depend on twelve parameters. In these published papers the authors studied many subclasses of quadratic systems. A list of them without trying to be exhaustive is the following: quadratic systems with a center [33, 38, 40, 52, 59]; with no finite real singularities $[17,50]$; with a unique finite singularity $[10,20,37,49,51,54,55]$; with a focus and one anti-saddle [2]; with an integrable saddle [6]; with a third order weak focus [5, 31]; with all points at infinity as singularities [18, 45]; that are Hamiltonian [3, 4, 22]), that are bounded [11, 26], that are Darboux [28, 53]; that are homogeneous $[56,57]$; that are Lotka-Volterra [47, 48]; structurally stable [1, 21]; semilinear [32]; with invariant lines of total multiplicity greater than or equal to four $[41,42,43,44,46]$; with rational first integrals [7, 29, 30]; with polynomial first integrals [16]; with a polynomial inverse integrating factor $[8], \ldots$

Nowadays there are still many open questions regarding quadratic systems. In this paper our objective is to characterize all the global phase portraits in the Poincaré disc of the class of quadratic systems which come from Abel quadratic polynomial differential equations of the second kind.

An Abel differential equation of the second kind is of the form

$$
y \frac{d y}{d x}=A(x) y+B(x),
$$

2010 Mathematics Subject Classification. Primary 37J35, 37K10.

Key words and phrases. Abel polynomial differential systems of the second kind, Vector fields, Phase portrait. 
with $A(x)$ and $B(x)$ non-zero functions, or equivalently, to the differential system

$$
\begin{aligned}
& \dot{x}=y c(x), \\
& \dot{y}=a(x) y+b(x),
\end{aligned}
$$

where $A(x)=a(x) / c(x)$ and $B(x)=b(x) / c(x)$. In this paper we are interested in studying the Abel quadratic polynomial differential systems, i.e. the differential systems of the form

$$
\begin{aligned}
& \dot{x}=y c(x):=y\left(c_{0}+c_{1} x\right), \\
& \dot{y}=a(x) y+b(x):=\left(a_{0}+a_{1} x\right) y+b_{0}+b_{1} x+b_{2} x^{2},
\end{aligned}
$$

where $a_{0}, a_{1}, b_{0}, b_{1}, b_{2}, c_{0}, c_{1} \in \mathbb{R}$ and such that $\dot{x}$ and $\dot{y}$ do not have a common factor, so $b_{0}^{2}+b_{1}^{2}+b_{2}^{2} \neq 0$. Moreover we assume that $c_{0}^{2}+c_{1}^{2} \neq 0$, otherwise the system is trivial. Note that we always have $a_{0}^{2}+a_{1}^{2} \neq 0$, otherwise would not be an Abel equation of the second kind. Moreover we additionally have that $b_{2}^{2}+a_{1}^{2}+c_{1}^{2} \neq 0$ in order that the system be a quadratic system.

Note that systems (2) have seven parameters and, at present, the full classification of their global phase portraits is unabordable. So we restrict to the ones that have a $\mathbb{Z}_{2}$-symmetry. We recall that there are two types of $\mathbb{Z}_{2}$-symmetric systems: the equivariant and the reversible ones. Let $\mathcal{X}: U \subset$ $\mathbb{R}^{2} \rightarrow \mathbb{R}^{2}$ be the vector field associated to system (2). We say that it is equivariant if either

$$
\left(\begin{array}{cc}
-1 & 0 \\
0 & 1
\end{array}\right) \mathcal{X}(x, y)=\mathcal{X}(-x, y)
$$

or

$$
\left(\begin{array}{cc}
1 & 0 \\
0 & -1
\end{array}\right) \mathcal{X}(x,-y)=\mathcal{X}(x,-y),
$$

and $\mathcal{X}$ is reversible if either

$$
\left(\begin{array}{cc}
-1 & 0 \\
0 & 1
\end{array}\right) \mathcal{X}(x, y)=-\mathcal{X}(-x, y)
$$

or

$$
\left(\begin{array}{cc}
1 & 0 \\
0 & -1
\end{array}\right) \mathcal{X}(x,-y)=-\mathcal{X}(x,-y) .
$$

Different families of planar polynomial vector fields with a $\mathbb{Z}_{2}$-symmetry have been studied by several authors, see for instance [24, 27, 58].

Note that by this definition system (2) is equivariant satisfying (3) if and only if $c_{1}=a_{0}=b_{0}=b_{2}=0$ and in this case system (2) becomes

$$
\begin{aligned}
& \dot{x}=c_{0} y, \\
& \dot{y}=a_{1} x y+b_{1} x,
\end{aligned}
$$

where $a_{1} b_{1} c_{0} \neq 0$.

System (2) is equivariant satisfying (4) if and only if $c_{0}=c_{1}=b_{0}=b_{1}=$ $b_{2}=0$ which is not possible. 
System (2) is reversible satisfying (5) if and only if $c_{0}=a_{1}=b_{1}=0$ and system (2) becomes

$$
\begin{aligned}
& \dot{x}=c_{1} y x, \\
& \dot{y}=a_{1} x y+b_{0}+b_{2} x^{2},
\end{aligned}
$$

where $a_{1} c_{1} b_{0} \neq 0$.

Finally system (2) is reversible satisfying (6) if and only if $a_{0}=a_{1}=0$ which is again not possible.

In this work we provide the global phase portraits of the Abel quadratic polynomial differential systems of the second kind that are equivariant (i.e. system (7)), and that are reversible (i.e. system (8)). For doing this we will use the Poincaré compactification of polynomial vector fields, see the Appendix.

We say that two polynomial vector fields in the Poincaré disk are topologically equivalent if there exists a homeomorphism from one onto the other which sends orbits to orbits preserving or reversing the direction of the flow.

Theorem 1. All quadratic polynomial differential systems (7), after a linear change of variables and a rescaling of its independent variable $t$, can be written as one of the following systems:

(I.1) $\dot{x}=y, \dot{y}=x(y+1)$;

(I.2) $\dot{x}=y, \dot{y}=x(y-1)$.

The global phase portrait for system (I.1) and (I.2) is topologically equivalent to (10) and (11) of Figure 1, respectively.

The proof of Theorem 1 is given in section 2 .

Theorem 2. All quadratic polynomial differential systems (8), after a linear change of variables and a rescaling of its independent variable $t$, can be written as one of the following five classes:

(II.1) $\dot{x}=x y, \dot{y}=\mu+x y$ with $\mu \in\{-1,1\}$;

(II.2) $\dot{x}=x y, \dot{y}=1+x^{2}+a x y$;

(II.3) $\dot{x}=x y, \dot{y}=-1-x^{2}+a x y$;

(II.4) $\dot{x}=x y, \dot{y}=-1+x^{2}+a x y$;

(II.5) $\dot{x}=x y, \dot{y}=1-x^{2}+a x y$;

where $a \in \mathbb{R}$. Moreover the global phase portrait for

- system (II.1) with $\mu=-1$ is (1),

- system (II.1) with $\mu=1$ is (2),

- system (II.2) is (2),

- system (II.3) with $|a|>2$ is (1),

- system (II.3) with $|a|=2$ is (3),

- system (II.3) with $|a|<2$ is (4), 


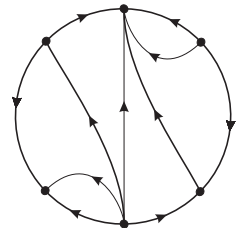

(1)

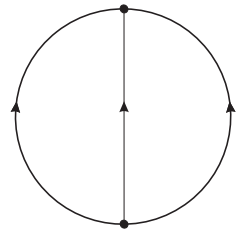

(4)

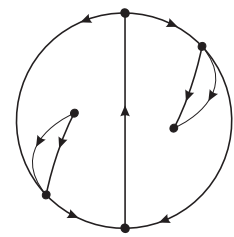

(7)

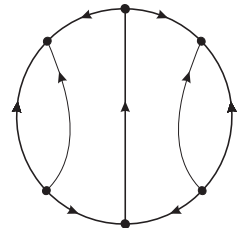

(2)

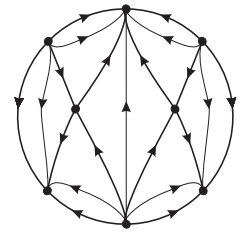

(5)

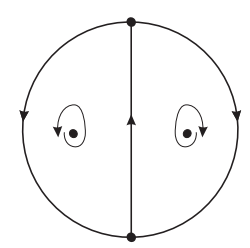

(8)

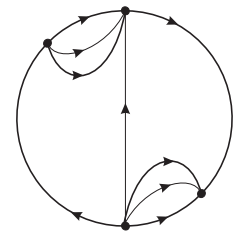

(3)

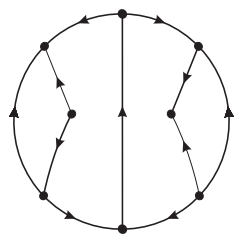

(6)

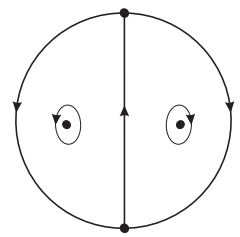

(9)

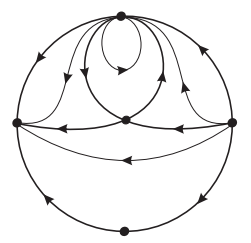

(10)

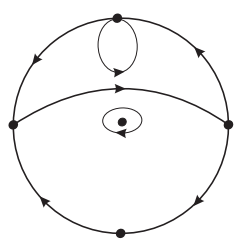

(11)

FiguRE 1. The global phase portraits in the Poincaré disc for the Abel quadratic polynomial differential equations of second kind with $\mathbb{Z}_{2}$-symmetries.

- system (II.4) is (5),

- system (II.5) with $|a|>2$ is (6),

- system (II.5) with $|a|=2$ is (7),

- system (II.5) with $|a|<2$ and $a \neq 0$ is (8),

- system (II.5) with $a=0$ is (9),

of Figure 1.

In section 3 we prove Theorem 2. We have an appendix where we provide some preliminary definitions, notations and theorems about the Poincaré sphere and the Poincaré compactification as well as results on canonical regions that we shall use for proving Theorems 1 and 2 . 
For more information on the phase portraits of Figure 1 see Table 1 and the proof of Theorem 1.

\section{Proof of Theorem 1}

We first prove the normal form provided in Theorem 1. To do that we consider the rescaling of variables

$$
x \rightarrow \delta X, \quad y \rightarrow \beta Y, \quad t \rightarrow \gamma T,
$$

with $\beta \delta \gamma \neq 0$. Note that $b_{1} c_{0} \neq 0$. If $b_{1} c_{0}>0$ we take

$$
\delta=\frac{a_{1}}{\sqrt{b_{1} c_{0}}}, \quad \beta=\frac{a_{1}}{b_{1}}, \quad \gamma=\sqrt{b_{1} c_{0}},
$$

obtaining system (I.1). If $b_{1} c_{0}<0$ we take

$$
\delta=\frac{a_{1}}{\sqrt{\left|b_{1} c_{0}\right|}}, \quad \beta=-\frac{a_{1}}{b_{1}}, \quad \gamma=\sqrt{\left|b_{1} c_{0}\right|},
$$

obtaining system (I.2).

Now we study the local phase portraits of the finite and infinite singular points of system (I.1).

The origin is the unique finite singular point which is a hyperbolic saddle because the eigenvalues of the Jacobian matrix at the origin are \pm 1 .

On the local chart $U_{1}$ (see the Appendix) system (I.1) becomes

$$
\dot{u}=u+v-u^{2} v, \quad \dot{v}=-u v^{2} .
$$

The unique singular point on $v=0$ is the origin $w_{0}=(0,0)$ which is semihyperbolic because the eigenvalues of the Jacobian matrix at $w_{0}$ are 1 and 0 . Using [15, Theorem 2.19] we get that it is a semi-hyperbolic unstable node.

On the local chart $U_{2}$ (see again the Appendix) system (I.1) becomes

$$
\dot{u}=v-u^{2}-u^{2} v, \quad \dot{v}=-u v-u v^{2} .
$$

The origin of the local chart $U_{2}$ is nilpotent. Using [15, Theorem 3.5] we obtain that it is locally the union of one elliptic, two parabolic and one hyperbolic sectors. Applying blow-up techniques we conclude that the hyperbolic sector is separated from the others by the infinity.

Using this information on the local phase portraits of the finite and infinite singular points, that $y=-1$ is an invariant straight line of the system, and that it is symmetric with respect to the $y$-axis we conclude that the unique possible global phase portrait is topologically equivalent to (10) in Figure 1. Moreover using the Appendix we conclude that the global phase portrait (10) has 4 canonical regions and 13 separatrices.

Now we study the local phase portraits of the finite and infinite singular points of system (I.2). 
The origin is the unique finite singular point which is a center because the eigenvalues of the Jacobian matrix at the origin are $\pm i$ and the system has the analytic first integral $H=(y-1)^{2} e^{2 y-x^{2}}$.

On the local chart $U_{1}$ system (I.2) becomes

$$
\dot{u}=u-v-u^{2} v, \quad \dot{v}=-u v^{2} .
$$

The unique singular point on $v=0$ is the origin $w_{0}=(0,0)$ which is semihyperbolic because the eigenvalues of the Jacobian matrix at $w_{0}$ are 1 and 0 . Using [15, Theorem 2.19] we get that it is a semi-hyperbolic saddle.

On the local chart $U_{2}$ system (I.2) becomes

$$
\dot{u}=v-u^{2}+u^{2} v, \quad \dot{v}=-u v+u v^{2} .
$$

The origin of the local chart $U_{2}$ is nilpotent. Using [15, Theorem 3.5] we get that it is the union of one elliptic sector and one hyperbolic sector. Applying blow-up techniques we conclude that both sectors are separated by the infinity.

Using this information on the local phase portraits of the finite and infinite singular point, that $y=1$ is an invariant straight line of the system, and that it is symmetric with respect to the $y$-axis we conclude that the unique possible global phase portrait is topologically equivalent to (11) in Figure 1. Finally, using the Appendix we conclude that the phase portrait has 2 canonical regions and 10 separatrices.

\section{Proof of Theorem 2}

We consider the linear change of variables and a rescaling of the independent variable (the time) of the form

$$
x \rightarrow \delta X, \quad y \rightarrow \beta Y, \quad t \rightarrow \gamma T,
$$

with $\beta \delta \gamma \neq 0$. Since $c_{1} \neq 0$ we take

$$
\gamma=\frac{1}{c_{1} \beta}
$$

and so we always have $X^{\prime}=X Y$.

If $b_{2}=0$ then since $a_{1} b_{0} \neq 0$ we take

$$
\beta=\sqrt{\left|\frac{b_{0}}{c_{1}}\right|} \text { and } \delta=\frac{c_{1} \beta}{a_{1}} .
$$

Then $Y^{\prime}=\mu+X Y$ with $\mu \in\{-1,1\}$, and we obtain the normal form (II.1) of the theorem.

If $b_{2} \neq 0$, since $b_{0} \neq 0$, we take

$$
\beta=\sqrt{\left|\frac{b_{0}}{c_{1}}\right|} \text { and } \delta=\sqrt{\left|\frac{b_{0}}{b_{2}}\right|} .
$$


Then $Y^{\prime}=\mu+\nu X^{2}+a X Y$ with $\mu, \nu \in\{-1,1\}$ and $a \in \mathbb{R}$. We obtain the normal forms (II.2)-(II.5) of Theorem 2.

Systems (II.1)-(II.5) have the invariant straight line $F=x=0$, and so they have at most one limit cycle (see, for instance, $[9,12]$ ).

Now we study the finite singular points of systems (II.1)-(II.5).

Systems (II.1), (II.2) and (II.3) have no finite singular points.

System (II.4) has the finite singular points $p_{0}=(1,0)$ and $p_{1}=(-1,0)$. Both of them are hyperbolic saddles because the eigenvalues are $\left(a \pm \sqrt{a^{2}+8}\right) / 2$ for $p_{0}$ and $\left(-a \pm \sqrt{a^{2}+8}\right) / 2$ for $p_{1}$, for all $a \in \mathbb{R}$.

System (II.5) has the finite singular points $p_{0}=(1,0)$ and $p_{1}=(-1,0)$. The eigenvalues at $p_{0}$ are $\left(a \pm \sqrt{a^{2}-8}\right) / 2$, and the eigenvalues at $p_{1}$ are $\left(-a \pm \sqrt{a^{2}-8}\right) / 2$. If $a=0$ then $p_{0}$ and $p_{1}$ are centers (the system has the analytic first integral $\left.H=x^{2} e^{-\left(x^{2}+y^{2}\right)}\right)$. If $a \neq 0$ and $a^{2} \geq 8$ then $p_{0}$ and $p_{1}$ are hyperbolic nodes: $p_{0}$ is stable if $a<0$ and unstable if $a>0$, while $p_{1}$ is stable if $a>0$ and unstable if $a<0$. If $a \neq 0$ and $a^{2}<8$, then $p_{0}$ and $p_{1}$ are foci: $p_{0}$ is stable if $a<0$ and unstable if $a>0$, while $p_{1}$ is stable if $a>0$ and unstable if $a<0$.

Finally we study the local phase portraits of the infinite singular points.

System (II.1). In the local chart $U_{1}$ system (II.1) becomes

$$
\dot{u}=u-u^{2}+\mu v^{2}, \quad \dot{v}=-u v .
$$

On $v=0$ we have the singular points $w_{0}=(0,0)$ and $w_{1}=(1,0)$. The singular point $w_{1}$ is a hyperbolic stable node because the eigenvalues of the Jacobian matrix at $w_{1}$ are -1 and -1 . The singular point $w_{0}$ is semihyperbolic because the eigenvalues of the Jacobian matrix at $w_{0}$ are 1 and 0 . Using [15, Theorem 2.19] we get that it is a saddle if $\mu=-1$, and an unstable node if $\mu=1$.

On the local chart $U_{2}$ system (I) becomes

$$
\dot{u}=u\left(1-u-\mu v^{2}\right), \quad \dot{v}=-v\left(u+\mu v^{2}\right) .
$$

The origin, $w_{2}$ is a singular point. The eigenvalues of the Jacobian matrix at the origin are $\lambda_{1}=1$ and $\lambda_{2}=0$. So, it is semi-hyperbolic. Using [15, Theorem 2.19] we get that it is a saddle if $\mu=1$, and an unstable node if $\mu=-1$.

System (II.2). In the local chart $U_{1}$ we have

$$
\dot{u}=1+a u-u^{2}+v^{2}, \quad \dot{v}=-u v
$$

There are two singular points $\left.w_{0}=\left(a+\sqrt{4+a^{2}}\right) / 2,0\right)$ and $w_{1}=(a-$ $\left.\left.\sqrt{4+a^{2}}\right) / 2,0\right)$. The singular point $w_{0}$ is a hyperbolic stable node because the eigenvalues of the Jacobian matrix at $w_{0}$ are $-\sqrt{4+a^{2}}$ and $-(a+$ 
$\left.\sqrt{4+a^{2}}\right) / 2$. The singular point $w_{1}$ is a hyperbolic unstable node because the eigenvalues of the Jacobian matrix at $w_{1}$ are $\sqrt{4+a^{2}}$ and $-\left(a-\sqrt{4+a^{2}}\right) / 2$.

On the local chart $U_{2}$ we have

$$
\dot{u}=u\left(1-a u-u^{2}-v^{2}\right), \quad \dot{v}=-v\left(a u+u^{2}+v^{2}\right) .
$$

The origin of $U_{2}$ is a singular point which is semi-hyperbolic because the eigenvalues at the origin $w_{2}$ are 1 and 0 . Using [15, Theorem 2.19] we get that it is a saddle.

System (II.3). In the local chart $U_{1}$ we have

$$
\dot{u}=-1+a u-u^{2}-v^{2}, \quad \dot{v}=-u v .
$$

There are two singular points $\left.w_{0}=\left(a+\sqrt{a^{2}-4}\right) / 2,0\right)$ and $w_{1}=(a-$ $\left.\left.\sqrt{a^{2}-4}\right) / 2,0\right)$ if and only if $|a|>2$. If $|a|=2$ then there is a unique singular point $w=w_{0}=w_{1}=(a / 2,0)$. When $|a|>2$, the singular point $w_{0}$ is a hyperbolic saddle if $a<0$ and a hyperbolic stable node if $a>0$ because the eigenvalues of the Jacobian matrix at $w_{0}$ are $-\sqrt{a^{2}-4}$ and $-\left(a+\sqrt{a^{2}-4}\right) / 2$. The singular point $w_{1}$ is a hyperbolic saddle if $a>0$ and a hyperbolic unstable node if $a<0$ because the eigenvalues of the Jacobian matrix at $w_{1}$ are $\sqrt{a^{2}-4}$ and $-\left(a-\sqrt{a^{2}-4}\right) / 2$. When $|a|=2$, the singular point $w$ is semi-hyperbolic. Using [15, Theorem 2.19] we get that it is a saddle-node.

On the local chart $U_{2}$ we have

$$
\dot{u}=u\left(1-a u+u^{2}+v^{2}\right), \quad \dot{v}=-v\left(a u-u^{2}-v^{2}\right) .
$$

The origin of $U_{2}$ is a singular point which is semi-hyperbolic because the eigenvalues at the origin $w_{2}$ are 1 and 0 . Using [15, Theorem 2.19] we get that it is an unstable node.

System (II.4). In the local chart $U_{1}$ we have

$$
\dot{u}=1+a u-u^{2}-v^{2}, \quad \dot{v}=-u v .
$$

There are two singular points $\left.w_{0}=\left(a+\sqrt{a^{2}+4}\right) / 2,0\right)$ and $w_{1}=(a-$ $\left.\left.\sqrt{a^{2}+4}\right) / 2,0\right)$. The singular point $w_{0}$ is a hyperbolic stable node because the eigenvalues of the Jacobian matrix at $w_{0}$ are $-\sqrt{4+a^{2}}$ and $-(a+$ $\left.\sqrt{4+a^{2}}\right) / 2$. The singular point $w_{1}$ is a hyperbolic unstable node because the eigenvalues of the Jacobian matrix at $w_{1}$ are $\sqrt{4+a^{2}}$ and $-\left(a-\sqrt{4+a^{2}}\right) / 2$.

On the local chart $U_{2}$ we have

$$
\dot{u}=u\left(1-a u-u^{2}+v^{2}\right), \quad \dot{v}=-v\left(a u+u^{2}-v^{2}\right) .
$$

The origin of $U_{2}$ is a singular point which is semi-hyperbolic because the eigenvalues at the origin $w_{2}$ are 1 and 0 . Using [15, Theorem 2.19] we get that it is an unstable node. 
System (II.5). In the local chart $U_{1}$ we have

$$
\dot{u}=-1+a u-u^{2}+v^{2}, \quad \dot{v}=-u v .
$$

There are two singular points $\left.w_{0}=\left(a+\sqrt{a^{2}-4}\right) / 2,0\right)$ and $w_{1}=(a-$ $\left.\left.\sqrt{a^{2}-4}\right) / 2,0\right)$ if and only if $|a|>2$. If $|a|=2$ then there is a unique singular point $w=w_{0}=w_{1}=(a / 2,0)$. When $|a|>2$, the singular point $w_{0}$ is a hyperbolic saddle if $a<0$ and a hyperbolic stable node if $a>0$ because the eigenvalues of the Jacobian matrix at $w_{0}$ are $-\sqrt{a^{2}-4}$ and $-\left(a+\sqrt{a^{2}-4}\right) / 2$. The singular point $w_{1}$ is a hyperbolic saddle if $a>0$ and a hyperbolic unstable node if $a<0$ because the eigenvalues of the Jacobian matrix at $w_{1}$ are $\sqrt{a^{2}-4}$ and $-\left(a-\sqrt{a^{2}-4}\right) / 2$. When $|a|=2$, the singular point $w$ is semi-hyperbolic. Using [15, Theorem 2.19] we get that it is a saddle-node.

On the local chart $U_{2}$ we have

$$
\dot{u}=u\left(1-a u+u^{2}-v^{2}\right), \quad \dot{v}=-v\left(a u-u^{2}+v^{2}\right) .
$$

The origin of $U_{2}$ is a singular point which is semi-hyperbolic because the eigenvalues at the origin $w_{2}$ are 1 and 0 . Using [15, Theorem 2.19] we get that it is a saddle.

In Table 1 we include all the information and phase portraits for the different systems (II.1)-(II.5) depending on their parameters. In this table we have the following notation: F.S.P. states for finite singular points, I.S.P. states for infinite singular points, P.P. states for phase portrait, C.R. states for canonical regions, $\Sigma$ states for sepatrices, $\mathrm{S}$ states for saddle, UN states for unstable node, SN states for stable node, UF states for unstable focus, $\mathrm{SF}$ states for stable focus, $\mathrm{C}$ states for center and S-N states for saddle-node.

Using the information on the local phase portraits described in Table 1 for the finite and infinite singular points, the existence of the invariant straight line $x=0$ (and consequently the system has at most one limit cycle), and the fact that whenever the local phase portrait has two foci it does not have limit cycles due to the symmetry with respect to the $y$-axis, each local phase portrait give rise to a unique global phase portrait in the Poincaré disc and we obtain nine topologically different global phase portraits as given in Table 1 and provided in Figure 1. Moreover for each global phase portrait using the results of the Appendix in Table 1 we describe the number of their canonical regions and separatrices.

\section{APPENDIX}

Poincaré compactification. Let

$$
\mathcal{X}=P(x, y) \frac{\partial}{\partial x}+Q(x, y) \frac{\partial}{\partial y}
$$


TABLE 1. Study of systems (II)

\begin{tabular}{|c||c|c|c|c|c|c|}
\hline Family & Conditions & F.S.P. & I.S.P. & P.P. & C.R. & $\Sigma$ \\
\hline (II.1) & $\mu=-1$ & $\emptyset$ & $w_{0}=\mathrm{S}, w_{1}=\mathrm{SN}, w_{2}=\mathrm{UN}$ & $(1)$ & 3 & 14 \\
\hline (II.1) & $\mu=1$ & $\emptyset$ & $w_{0}=\mathrm{UN}, w_{1}=\mathrm{SN}, w_{2}=\mathrm{S}$ & $(2)$ & 2 & 13 \\
\hline (II.2) & $a \in \mathbb{R}$ & $\emptyset$ & $w_{0}=\mathrm{SN}, w_{1}=\mathrm{UN}, w_{2}=\mathrm{S}$ & $(2)$ & 2 & 13 \\
\hline (II.3) & $a>2$ & $\emptyset$ & $w_{0}=\mathrm{SN}, w_{1}=\mathrm{S}, w_{2}=\mathrm{UN}$ & $(1)$ & 3 & 14 \\
\hline (II.3) & $a= \pm 2$ & $\emptyset$ & $w=\mathrm{S}-\mathrm{N}, w_{2}=\mathrm{UN}$ & $(3)$ & 3 & 10 \\
\hline (II.3) & $a \in(-2,2)$ & $\emptyset$ & $w_{2}=\mathrm{UN}$ & $(4)$ & 1 & 4 \\
\hline (II.3) & $a<-2$ & $\emptyset$ & $w_{0}=\mathrm{S}, w_{1}=\mathrm{UN}, w_{2}=\mathrm{UN}$ & $(1)$ & 3 & 14 \\
\hline (II.4) & $a \in \mathbb{R}$ & $p_{0}=\mathrm{S}, p_{1}=\mathrm{S}$ & $w_{0}=\mathrm{SN}, w_{1}=\mathrm{UN}, w_{2}=\mathrm{UN}$ & $(5)$ & 7 & 22 \\
\hline (II.5) & $a \geq 2 \sqrt{2}$ & $p_{0}=\mathrm{UN}, p_{1}=\mathrm{SN}$ & $w_{0}=\mathrm{SN}, w_{1}=\mathrm{S}, w_{2}=\mathrm{S}$ & $(6)$ & 2 & 17 \\
\hline (II.5) & $a \in(2,2 \sqrt{2})$ & $p_{0}=\mathrm{UF}, p_{1}=\mathrm{SF}$ & $w_{0}=\mathrm{SN}, w_{1}-\mathrm{S}, w_{2}=\mathrm{S}$ & $(6)$ & 2 & 17 \\
\hline (II.5) & $a=2$ & $p_{0}=\mathrm{UF}, p_{1}=\mathrm{SF}$ & $w=\mathrm{S}-\mathrm{N}, w_{2}=\mathrm{S}$ & $(7)$ & 2 & 13 \\
\hline (II.5) & $a \in(0,2)$ & $p_{0}=\mathrm{UF}, p_{1}=\mathrm{SF}$ & $w_{2}=\mathrm{S}$ & $(8)$ & 2 & 7 \\
\hline (II.5) & $a=0$ & $p_{0}=\mathrm{C}, p_{1}=\mathrm{C}$ & $w_{2}=\mathrm{S}$ & $(9)$ & 2 & 7 \\
\hline (II.5) & $a \in(-2,0)$ & $p_{0}=\mathrm{SF}, p_{1}=\mathrm{UF}$ & $w_{2}=\mathrm{S}$ & $(8)$ & 2 & 7 \\
\hline (II.5) & $a=-2$ & $p_{0}=\mathrm{SF}, p_{1}=\mathrm{UF}$ & $w=\mathrm{S}-\mathrm{N}, w_{2}=\mathrm{S}$ & $(7)$ & 2 & 13 \\
\hline (II.5) & $a \in(-2 \sqrt{2},-2)$ & $p_{0}=\mathrm{SF}, p_{1}=\mathrm{UF}$ & $w_{0}=\mathrm{S}, w_{1}=\mathrm{UN}, w_{2}=\mathrm{S}$ & $(6)$ & 2 & 17 \\
\hline (II.5) & $a \leq-2 \sqrt{2}$ & $p_{0}=\mathrm{SN}, p_{1}=\mathrm{UN}$ & $w_{0}=\mathrm{S}, w_{1}=\mathrm{UN}, w_{2}=\mathrm{S}$ & $(6)$ & 2 & 17 \\
\hline
\end{tabular}

be the quadratic polynomial vector field associated to the polynomial differential system (1). The Poincaré compactified vector field $p(\mathcal{X})$ associated to $\mathcal{X}$ is an analytic vector field on $\mathbb{S}^{2}$ constructed as follows (see, for instance [19], or Chapter 5 of [15]).

The Poincaré sphere is defined as $\mathbb{S}^{2}=\left\{y=\left(y_{1}, y_{2}, y_{3}\right) \in \mathbb{R}^{3}: y_{1}^{2}+y_{2}^{2}+\right.$ $\left.y_{3}^{2}=1\right\}$ and $T_{y} \mathbb{S}^{2}$ is the tangent plane to $\mathbb{S}^{2}$ at the point $y$. The plane $\mathbb{R}^{2}$ where we have our polynomial vector field $\mathcal{X}$ is identified with the tangent plane $T_{(0,0,1)} \mathbb{S}^{2}$. Now we consider the central projection $f: T_{(0,0,1)} \mathbb{S}^{2} \rightarrow \mathbb{S}^{2}$. It defines two copies of $\mathcal{X}$, one in the northern hemisphere and the other in the southern hemisphere. Denote by $\mathcal{X}^{\prime}$ the vector field $D f \circ \mathcal{X}$ defined on $\mathbb{S}^{2}$ except on $\mathbb{S}^{1}=\left\{y \in \mathbb{S}^{2}: y_{3}=0\right\}$. The equator $\mathbb{S}^{1}$ is identified to the infinity of $\mathbb{R}^{2}$. For extending $\mathcal{X}^{\prime}$ to a vector field on $\mathbb{S}^{2}$ (including $\mathbb{S}^{1}$ ) it is necessary that $\mathcal{X}$ satisfies suitable conditions. In our case $p(\mathcal{X})$ is the only analytic extension of $y_{3} \mathcal{X}^{\prime}$ to $\mathbb{S}^{2}$. On $\mathbb{S}^{2} \backslash \mathbb{S}^{1}$ there are two symmetric copies of $\mathcal{X}$, and knowing the behaviour of $p(\mathcal{X})$ around $\mathbb{S}^{1}$, we know the behaviour of $\mathcal{X}$ at infinity.

The Poincaré disc is just the projection of the closed northern hemisphere of $\mathbb{S}^{2}$ on $y_{3}=0$ under $\left(y_{1}, y_{2}, y_{3}\right) \longmapsto\left(y_{1}, y_{2}\right)$. It is denoted by $\mathbb{D}^{2}$. We note that the Poincaré compactification has the property that $\mathbb{S}^{1}$ is invariant under the flow of $p(\mathcal{X})$.

As we already stated in the introduction, we say that two polynomial vector fields $\mathcal{X}$ and $\mathcal{Y}$ on $\mathbb{R}^{2}$ are topologically equivalent if there exists a homeomorphism on $\mathbb{S}^{2}$ preserving the infinity $\mathbb{S}^{1}$ carrying orbits of the flow 
induced by $p(\mathcal{X})$ into orbits of the flow induced by $p(\mathcal{Y})$, preserving or reversing simultaneously the direction of all orbits.

As $\mathbb{S}^{2}$ is a differentiable manifold, in order to compute $p(\mathcal{X})$, we can consider the six local charts $U_{i}=\left\{y \in \mathbb{S}^{2}: y_{i}>0\right\}, V_{i}=\left\{y \in \mathbb{S}^{2}\right.$ : $\left.y_{i}<0\right\}$ for $i=1,2,3$; and the diffeomorphisms $F_{i}: U_{i} \rightarrow \mathbb{R}^{2}, G_{i}: V_{i} \rightarrow$ $\mathbb{R}^{2}$ for $i=1,2,3$ (here $G_{i}$ are the inverses of the central projections from the planes tangent at the points $(1,0,0),(-1,0,0),(0,1,0),(0,-1,0),(0,0,1)$ and $(0,0,-1)$ respectively). If we denote by $(u, v)$ the value of $F_{i}(y)$ or $G_{i}(y)$ for any $i=1,2,3$ (here $(u, v)$ represents different things according to the local charts under consideration), then some easy computations give for $p(\mathcal{X})$ the following expressions:

$$
\begin{array}{rc}
v^{2} \Delta(u, v)\left(Q\left(\frac{1}{v}, \frac{u}{v}\right)-u P\left(\frac{1}{v}, \frac{u}{v}\right),-v P\left(\frac{1}{v}, \frac{u}{v}\right)\right) & \text { in } U_{1}, \\
v^{2} \Delta(u, v)\left(P\left(\frac{u}{v}, \frac{1}{v}\right)-u Q\left(\frac{u}{v}, \frac{1}{v}\right),-v Q\left(\frac{u}{v}, \frac{1}{v}\right)\right) & \text { in } U_{2}, \\
\Delta(u, v)(P(u, v), Q(u, v)) & \text { in } U_{3},
\end{array}
$$

where $\Delta(u, v)=\left(u^{2}+v^{2}+1\right)^{-\frac{1}{2}}$. The expression for $V_{i}$ is the same as that for $U_{i}$ except for a multiplicative factor -1 . In these coordinates for $i=1,2$, $v=0$ always denotes the points of $\mathbb{S}^{1}$. In what follows we omit the factor $\Delta(u, v)$ by rescaling the vector field $p(\mathcal{X})$. Thus we obtain a polynomial vector field in each local chart.

Separatrices and canonical regions. We continue to denote by $p(\mathcal{X})$ the Poincaré compactification in the Poincaré disc $\mathbb{D}$ of the polynomial differential system (1), and by $\Phi$ its analytic flow. We follow the notation in Markus [34] and Neumann [35] and we let $(U, \Phi)$ be the flow of a differential system on an invariant set $U \subset \mathbb{D}$ under the flow $\Phi$. As before, two flows $(U, \Phi)$ and $(V, \Psi)$ are topologically equivalent if and only if there exists a homeomorphism $h: U \rightarrow V$ which sends orbits of the flow $\Phi$ into orbits of the flow $\Psi$ either preserving or reversing the orientation of all the orbits.

The flow $(U, \Phi)$ is parallel if it is topologically equivalent to one of the following flows:

(i) The flow defined in $\mathbb{R}^{2}$ by the differential system $\dot{x}=1, \dot{y}=0$, called strip flow.

(ii) The flow defined in $\mathbb{R}^{2} \backslash\{(0,0)\}$ by the differential system in polar coordinates $\dot{r}=0, \dot{\theta}=1$, called annular flow.

(iii) The flow defined in $\mathbb{R}^{2} \backslash\{(0,0)\}$ by the differential system in polar coordinates $\dot{r}=r, \dot{\theta}=0$, called spiral or radial flow.

It is well-known that the separatrices of the vector field $p(\mathcal{X})$ in the Poincaré disc $\mathbb{D}$ are: 
(I) all orbits of $p(\mathcal{X})$ which are in the boundary $\mathbb{S}^{1}$ of the Poincaré disc,

(II) all finite singular points of $p(\mathcal{X})$,

(III) all limit cycles of $p(\mathcal{X})$, and

(IV) all separatrices of the hyperbolic sectors of the finite and infinite singular points of $p(\mathcal{X})$.

Moreover such vector fields $p(\mathcal{X})$ have finitely many separatrices. See for instance [25] for further details.

We denote by $\mathcal{S}$ the union of the separatrices of the flow $(\mathbb{D}, \Phi)$ defined by $p(\mathcal{X})$ in the Poincaré disc $\mathbb{D}$. It is clear that $\mathcal{S}$ is an invariant closed set. If $N$ is a connected component of $\mathbb{D} \backslash \mathcal{S}$, then $N$ is also an invariant set under the flow $\Phi$ of $p(\mathcal{X})$, and we call the flow $\left(N,\left.\Phi\right|_{N}\right)$ a canonical region of $(\mathbb{D}, \Phi)$. For a proof of the following proposition see either [35] or [25].

Proposition 3. If the number of separatrices of the flow $(\mathbb{D}, \Phi)$ is finite, then every canonical region of the flow $(\mathbb{D}, \Phi)$ is parallel.

The separatrix configuration $\mathcal{S}_{c}$ of a flow $(\mathbb{D}, \Phi)$ is the union of all the separatrices $\mathcal{S}$ of the flow jointly with an orbit belonging to each canonical region. The separatrix configuration $\mathcal{S}_{c}$ of the flow $(\mathbb{D}, \Phi)$ is topologically equivalent to the separatrix configuration $\mathcal{S}_{c}^{*}$ of the flow $\left(\mathbb{D}, \Phi^{*}\right)$ if there exists an orientation preserving homeomorphism from $\mathbb{D}$ to $\mathbb{D}$ which transforms orbits of $\mathcal{S}_{c}$ into orbits of $\mathcal{S}_{c}^{*}$, and orbits of $\mathcal{S}$ into orbits of $\mathcal{S}^{*}$.

Theorem 4 (Markus-Neumann-Peixoto Theorem). Let $(\mathbb{D}, \Phi)$ and $\left(\mathbb{D}, \Phi^{*}\right)$ be two compactified Poincaré flows with finitely many separatrices coming from two polynomial vector fields (1). Then they are topologically equivalent if and only if their separatrix configurations are topologically equivalent.

For a proof of this result we refer the reader to [34, 35, 39].

It follows from the previous theorem that in order to classify the phase portraits in the Poincaré disc of a planar polynomial differential system having finitely many separatrices finite and infinite, it is enough to describe their separatrix configuration.

\section{ACKNOWLEDGEMENTS}

The first author is partially supported by a MINECO grant number MTM2013-40998-P, an AGAUR grant number 2014SGR-568 and the grants FP7-PEOPLE-2012-IRSES 318999 and 316338. The second author is partially supported by FCT/Portugal through UID/MAT/04459/2013.

\section{REFERENCES}

[1] J.C. Artés, R.E. Kooij and J. Llibre, Structurally stable quadratic vector fields, Mem. Amer. Math. Soc. 134 (1998), 108 pp. 
[2] J.C. Artés and J. Llibre, Phase portraits for quadratic systems having a focus and one antisaddle, Rocky Mountain J. Math. 24 (1994), 875-889.

[3] J.C. Artés and J. Llibre, Quadratic Hamiltonian vector fields, J. Differential Equations 107 (1994), 80-95.

[4] J.C. Artés and J. Llibre, Corregidum: A correction to the paper "Quadratic Hamiltonian vector fields" [J. Differential Equations 107 (1994), 80-95], J. Differential Equations 129 (1996), 559-560.

[5] J.C. Artés and J. Llibre, Quadratic vector fields with a weak focus of third order, Publicacions Matematiques 41 (1997), 7-39.

[6] J.C. Artés, J. Llibre and N. Vulpe, Quadratic systems with an integrable saddle: a complete classification in the coefficient space $\mathbb{R}^{12}$, Nonlinear Anal. 75 (2012), 54165447.

[7] L. Cairó and J. Llibre, Phase portraits of quadratic polynomial vector fields having a rational first integral of degree 2, Nonlinear Anal. 67 (2007), 327-348.

[8] B. Coll, A. Ferragut and J. Llibre, Phase portraits of the quadratic systems with a polynomial inverse integrating factor, Internat. J. Bifur. Chaos Appl. Sci. Engrg. 19 (2009), 765-783.

[9] B. Coll and J. Llibre, Limit cycles for a quadratic system with an invariant straight line and some evolution of phase portraits, in Qualitative Theory of Differential Equations, Colloquia Mathematica Societatis János Bolyai A 53 (1988), 111-123, Bolyai Institut, Szeged, Hungria.

[10] B. Coll, A. Gasull and J. Llibre, Quadratic systems with a unique finite rest point, Publicacions Matematiques 32 (1988), 199-259.

[11] B. Coll, A. Gasull and J. Llibre, Some theorems on the existence, uniqueness, and nonexistence of limit cycles for quadratic systems, J. Differential Equations 67 (1987), 372-399.

[12] W.A. Coppel, Some quadratic systems with at most one limit cycle, Dynamics reported, 2 (1989), 61-88, Dynam. Report. Ser. Dynam. Systems Appl., 2, Wiley, Chichester, 1989.

[13] T. Date, Classification and analysis of two-dimensional real homogeneous quadratic differential equation systems, J. Differential Equations 32 (1979), 311-334.

[14] F. Dumortier and C. Li, Quadratic Liénard equations with quadratic damping, J. Differential Equations 139 (1997), 41-59.

[15] F. Dumortier, J. Llibre and J. C. Artés, Qualitative Theory of Planar Differential Systems, UniversiText, Springer-verlag, New York, 2006.

[16] B. García, J. Llibre and J.S. Pérez del Río, Phase portraits of the quadratic vector fields with a polynomial first integral, Rend. Circ. Mat. Palermo 55 (2006), 420-440.

[17] A. Gasull, S. Li-Ren and J. Llibre, Chordal quadratic systems, Rocky Mountain J. Math 16 (1996), 751-782.

[18] A. Gasull and R. Prohens, Quadratic and cubic systems with degenerate infinity, J. Math. Anal. Appl. 198 (1996), 25-34.

[19] E.A. González, Generic properties of polynomial vector fields at infinity, Trans. Amer. Math. Soc. 143 (1969), 201-222.

[20] P. De Jager, Phase portraits for quadratic systems with higher order singularity with two zero eigenvalues, J. Differential Equations 87 (1990), 169-204.

[21] X. Jarque, J. Llibre and D.S. Shafer, Structurally stable quadratic foliations, Rocky Mountain J. Math. 38 (2008), 489-530.

[22] Yu.F. Kalin and N.I. Vulpe, Affine-invariant conditions for the topological discrimination of quadratic Hamiltonian differential systems, (Russian), Differ. Uravn. 34 (1998), 298-302, 428; translation in: Differential Equations 34 (1998), 297-301.

[23] C. Li, Two problems of planar quadratic systems, Scientia Sinica Series A 26 (1983), $471-481$. 
[24] J. Li and Y. Liu, Global bifurcation in a perturbed cubic system with $\mathbb{Z}_{2}$-symmetry, Acta Math. Appl. Sinica 8 (1992), 131-143.

[25] W. Li, J. Llibre, M. Nicolau and X. Zhang, On the differentiability of first integrals of two dimensional flows, Proc. Amer. Math. Soc. 130 (2002), 2079-2088.

[26] C.Z. Li, J. Llibre and Z.F. Zhang, Weak focus, limit cycles, and bifurcations for bounded quadratic systems, J. Differential Equations 115 (1995), 193-223.

[27] Y. Liu and J. Li, $\mathbb{Z}_{2}$-equivariant cubic system which yields 13 limit cycles, Acta Math. Appl. Sinica 30 (2014), 781-800.

[28] J. Llibre and J.C. Medrado, Darboux integrability and reversible quadratic vector fields, Rocky Mountain J. Math. 35 (2005), 1999-2057.

[29] J. Llibre and R.D.S. Oliveira, Phase portraits of quadratic polynomial vector fields having a rational first integral of degree 3, Nonlinear Anal. 70 (2009), 3549-3560.

[30] J. Llibre and R.D.S. Oliveira, Erratum to "Phase portraits of quadratic polynomial vector fields having a rational first integral of degree 3" [Nonlinear Anal. 70 (2009), 3549-3560], Nonlinear Anal. 71 (2009), 6378-6379.

[31] J. Llibre and D. Schlomiuk, The geometry of quadratic differential systems with a weak focus of third order, Canad. J. Math. 56 (2004), 310-343.

[32] J. Llibre and X. Zhang, Topological phase portraits of planar semi-linear quadratic vector fields, Houston J. Math. 27 (2001), 247-296.

[33] M. Lupan and N. Vulpe, Classification of quadratic systems with a symmetry center and simple infinite singular points. In memory of C.S. Sibirsky, Bul. Acad. Ştiinţe Repub. Mold. Mat. 2003, (2003), 102-119.

[34] L. Markus, Global structure of ordinary differential equations in the plane: Trans. Amer. Math Soc. 76 (1954), 127-148.

[35] D. A. Neumann, Classification of continuous flows on 2-manifolds, Proc. Amer. Math. Soc. 48 (1975), 73-81.

[36] D. Neumann, Classification of continuous flows on 2-manifolds, Proc. Amer. Math. Soc. 48 (1975), 73-81.

[37] I.V. Nikolaev and N.I. Vulpe, Topological classification of quadratic systems with a unique finite second order singularity with two zero eigenvalues, Izv. Akad. Nauk. Respub. Moldova Mat. 1993 (1993), 3-8, 107, 109.

[38] J. Pal and D. Schlomiuk, Summing up the dynamics of quadratic Hamiltonian systems with a center, Canad. J. Math. 49 (1997), 583-599.

[39] M.M. Peixoto, Dynamical Systems. Proccedings of a Symposium held at the University of Bahia, 389-420, Acad. Press, New York, 1973.

[40] D. Schlomiuk, Algebraic particular integrals, integrability and the problem of the center, Trans. Amer. Math. Soc. 338 (1993), 799-841.

[41] D. Schlomiuk and N. Vulpe, Planar quadratic vector fields with invariant lines of total multiplicity at least five, Qual. Theory Dyn. Syst. 5 (2004), 135-194.

[42] D. Schlomiuk and N. Vulpe, Integrals and phase portraits of planar quadratic differential systems with invariant lines of at least five total multiplicity, Rocky Mountain J. Math. 38 (2008), 2015-2075.

[43] D. Schlomiuk and N. Vulpe, Planar quadratic differential systems with invariant straight lines of total multiplicity four, Nonlinear Anal. 68 (2008), 681-715.

[44] D. Schlomiuk and N. Vulpe, Integrals and phase portraits of planar quadratic differential systems with invariant lines of total multiplicity four, Bul. Acad. Ştiințe Repub. Mold. Mat. 2008 (2008), 27-83.

[45] D. Schlomiuk and N. Vulpe, The full study of planar quadratic systems possessing a line of singularities at infinity, J. Dynam. Differential Equations 20 (2008), 737-775.

[46] D. Schlomiuk and N. Vulpe, Bifurcation diagrams and moduli spaces of planar quadratic vector fields with invariant lines of total multiplicity four and having exactly three real singularities at infinity, Qual. Theory Dyn. Syst. 9 (2010), 251-300. 
[47] D. Schlomiuk and N. Vulpe, Global classification of the planar Lotka-Volterra differential systems according to their configurations of invariant straight lines, J. Fixed Point Theory Appl. 8 (2010), 177-245.

[48] D. Schlomiuk and N. Vulpe, Global topological classification of Lotka-Volterra quadratic differential systems, Electron. J. Differential Equations 2012 (2012), 69 pp.

[49] M. Voldman, Iu.T. Calin and V.I. Vulpe, Affine invariant conditions for the topological distinction of quadratic systems with a critical point of 4 th multiplicity, Publicacions Matematiques. 40 (1996), 431-441.

[50] M. Voldman and N.I. Vulpe, Affine invariant conditions for topologically distinguishing quadratic systems without finite critical points, Izv. Akad. Nauk. Respub. Moldova Mat. 1995 (1995), 100-112, 114, 117.

[51] M. Voldman and N.I. Vulpe, Affine invariant conditions for topologically distinguishing quadratic systems with $m_{f}=1$, Nonlinear Anal. 31 (1998), 171-179.

[52] N.I. Vulpe, Affine-invariant conditions for topological distinction of quadratic systems in the presence of a center, (Russian), Differentsial'nye Uravneniya 19 (1983), 371379.

[53] N.I. Vulpe and A.Yu. Likhovetskii, Coefficient conditions for the topological discriminant of quadratic systems of Darboux type, (Russian), Mat. Issled. 106, Differ. Uravneniya i Mat. Fizika (1989), 34-49, 178.

[54] N.I. Vulpe and I.V. Nikolaev, Topological classification of QS with a unique third order singular point, Izv. Akad. Nauk. Respub. Moldova Mat. 1992 (1992), 37-44.

[55] N.I. Vulpe and I.V. Nikolaev, Topological classification of quadratic systems with a four-fold singular point, (Russian), Differentsial'nye Uravneniya 29 (1993), 1669 1674, 1836; translation in: Differential Equations 29 (1993), 1449-1453 (1994).

[56] N.I. Vulpe and K.S. Sibirskii, Affinely invariant coefficient conditions for the topological distinctness of quadratic systems, (Russian), Mat. Issled. 10 (1975), 15-28, 238.

[57] N.I. Vulpe and K.S. Sibirskii, Geometric classification of a quadratic differential system, (Russian), Differentsial'nye Uravneniya 13 (1977), 803-814, 963.

[58] P. Yu and M. Han, On limit cycles of the Liénard equation with $\mathbb{Z}_{2}$ symmetry, Chaos, Solitons \& Fractals 31 (2007), 617-630.

[59] H. Zolądek, Quadratic systems with a center and their perturbations, J. Differential Equations 109 (1994), 223-273.

${ }^{1}$ Departament de Matemàtiques, Universitat Autònoma de Barcelona, 08193 Bellaterra, Barcelona, Catalonia, Spain

E-mail address: jllibre@mat.uab.cat

2 Departamento de Matemática, Instituto Superior Técnico, Universidade Técnica de Lisboa, Av. Rovisco Pais 1049-001, Lisboa, Portugal

E-mail address: cvalls@math.ist.utl.pt 